\title{
Atomic Mechanism of Carbon Diffusion in Cementite
}

\author{
A.V. Evteev ${ }^{1, a}$, E.V. Levchenko, ${ }^{1, b}$, I.V. Belova ${ }^{1, c}$ and G.E. Murch ${ }^{1, d}$ \\ ${ }^{1}$ Diffusion in Solids Group, School of Engineering, \\ The University of Newcastle, Callaghan, NSW 2308, Australia \\ aAlexander.Evteev@newcastle.edu.au, ${ }^{\mathrm{b}}$ Elena.Levchenko@newcastle.edu.au, \\ 'Irina.Belova@newcastle.edu.au, 'Graeme.Murch@newcastle.edu.au
}

\begin{abstract}
Keywords: Carbon diffusion, cementite, atomistic modelling, molecular dynamics.
\end{abstract}
\begin{abstract}
Molecular dynamics is employed to investigate carbon diffusion in cementite. An approximation that carbon atoms can interact with each other only indirectly (via neighbouring iron atoms) is used. The interstitial mechanism of carbon diffusion in cementite is elucidated. The formation energy of defects (a carbon atom on an interstitial position and a vacant site on a regular carbon position) as well as the migration energy of carbon atoms are estimated in the temperature range 1273-1373 K.
\end{abstract}

\section{Introduction}

Although cementite $\left(\mathrm{Fe}_{3} \mathrm{C}\right)$ is a most important phase in steels, at the same time, there is rather little known in regard to the fundamental properties of $\mathrm{Fe}_{3} \mathrm{C}$. This lack of information is largely a result of this compound being metastable with respect to its decomposition products: carbon saturated ferrite or austenite (depending on temperature) and graphite. Hence, it is difficult to obtain 'pure' $\mathrm{Fe}_{3} \mathrm{C}$ in the sizes and amounts necessary for many fundamental studies including investigation of its thermodynamic and diffusion properties [1-3].

In the last few years, apart from the technological importance of cementite itself, the role of cementite in the metal dusting process has attracted a great deal of interest. In the metal dusting process, strongly carbon-bearing gases with a carbon activity $>>1$ attack iron and low alloyed steels, converting them into micro and nanoparticulate dust [3-7]. The role reversal of the mobile species iron and carbon at different times in the metal dusting process, the central role of cementite and its metastability, the presence of local carbon thermodynamic activities that are seemingly sensitive to the state of solid carbon all combine to make the understanding of metal dusting from experiments alone very challenging. Conventional radiotracer diffusion experiments in cementite are essentially impossible and chemical diffusion in cementite is problematic in its experimental conditions. Furthermore, it is also difficult to determine the responsible mobile species and atomistic mechanism from such experiments.

It is appropriate therefore to make use of modern computer simulation techniques to elucidate some of the atomistic details of diffusion occurring in metal dusting. This paper describes a molecular dynamics study of the detailed mechanism of carbon diffusion in cementite and is the first part of a comprehensive computational/theoretical program.

\section{The Model}

The $\mathrm{Fe}_{3} \mathrm{C}$ structure was simulated as a calculation box with periodic boundary conditions consisting of $10 \times 10 \times 10$ simple orthorhombic unit cells with lattice parameters $a=4.523 \AA, b=5.089 \AA$ and $c=6.743 \AA$ and $4 \mathrm{Fe}$ atoms of type $1,8 \mathrm{Fe}$ atoms of type 2 and $4 \mathrm{C}$ atoms per unit cell [8]. Fe (1) and $\mathrm{Fe}(2)$ are two different types of iron sites. The atomic positions are listed in the Table 1 . Thus, this model contains $12000 \mathrm{Fe}$ atoms and $4000 \mathrm{C}$ atoms.

Table 1. The atomic positions in cementite in units of the lattice parameters $a, b$ and $c$, respectively.

\begin{tabular}{|c|c|c|}
\hline Axis & $\mathrm{Fe}(1)$ & $\mathrm{Fe}(2)$ \\
\hline$x_{1}$ & \begin{tabular}{l|l|l|l|l|}
33 & 0.167 & 0.6 \\
\end{tabular} & \begin{tabular}{|l|l|l|l|l|l|l|}
333 & 0.667 & 0.167 & 0.833 & 0.667 & 0 \\
\end{tabular} \\
\hline$x_{2}$ & 0.0400 .960 & \begin{tabular}{l|l|l}
5 & 0.6750.
\end{tabular} \\
\hline & 025007500 & \begin{tabular}{|l|l|l|l|l|l|l|l|l|l|l|l|}
0.065 & 0.935 & 0.435 & 0.565 & 0.565 & 0.435 & 0.935 & 0.065 & 0.250 & 0.750 \\
\end{tabular} \\
\hline
\end{tabular}


The Fe-Fe interaction was described by the well-known Johnson empirical pair-potential [9]:

$$
\varphi_{\mathrm{Fe}-\mathrm{Fe}}(r)= \begin{cases}-2.195976(r-3.097910)^{3}+2.704060 r-7.436448 \mathrm{eV}, & r<2.40 \AA, \\ -0.639230(r-3.115829)^{3}+0.477871 r-1.581570 \mathrm{eV}, & 2.40 \AA \leq r<3.00 \AA, \\ -1.115035(r-3.066403)^{3}+0.466892 r-1.547967 \mathrm{eV}, & 3.00 \AA \leq r \leq 3.44 \AA .\end{cases}
$$

The Johnson potential and its first derivative smoothly go to zero at a cut-off radius of $3.44 \AA$. The parameters for this potential have been chosen in such a way that the elastic and dynamic properties of $\alpha$-Fe were matched. This potential represents very well the behaviour of Fe interstitials, vacancies and their complexes in $\alpha-\mathrm{Fe}[9,10]$. Later, Johnson [11] showed that the same potential can be also used for $\gamma$-Fe and it permits an adequate description of the elastic and dynamic properties as well as the behaviour of the point defects and their complexes. A discussion and proof of the validity of such a seemingly arbitrary choice for the f.c.c. case is presented in [11].

To describe the Fe-C pair interaction we used the potential proposed by Johnson, Dienes and Damask [12]:

$$
\varphi_{\mathrm{Fe}-\mathrm{C}}(r)=-\varepsilon\left[2\left(\frac{r-r_{\mathrm{c}}}{r_{\mathrm{c}}-r_{0}}\right)^{3}+3\left(\frac{r-r_{\mathrm{c}}}{r_{\mathrm{c}}-r_{0}}\right)^{2}\right] .
$$

Fitting of the Johnson-Dienes-Damask potential to the experimental data for $\alpha$-Fe (the migration energy of carbon atoms, the activation volume of carbon migration and the vacancy-carbon binding energy) was performed in a set of computer experiments using the Fe-Fe Johnson potential (Eq. 1). Three critical parameters have been changed: the position $r_{0}$, the depth $\varepsilon$ of the potential well and the potential cut-off radius $r_{\mathrm{c}}$. The final values for these parameters are: $r_{0}=1.94 \AA, \varepsilon=0.35 \mathrm{eV}$, $r_{\mathrm{c}}=2.53 \AA$ [12]. The use of the Johnson-Dienes-Damask potential together with the Johnson potential allows for an adequate description of the behaviour of carbon interstitials in $\alpha$-Fe [12-14] and in martensite [15]. The structure and relative energy of cementite [12] have also been investigated by means of these potentials. The use of the Johnson-Dienes-Damask potential together with the empirical Pak-Doyama pair potential [16] (this is very similar to the Johnson potential) gives good agreement between the structural characteristics of the MD model of $\mathrm{Fe}-\mathrm{C}$ melts and experimental data [17]. At the same time, this combination of potentials provides an explanation of the low glassforming ability of the metallic alloys of this system compared to metallic Fe-B and Fe-P alloys [17]. More recently, the Johnson-Dienes-Damask potential has been used in a series of studies of carbon nanotube growth on the surface of an iron cluster; see, for example, [18].

The situation with the (weak) carbon-carbon potential is more complicated. There is no agreed and detailed data about the preferred type of direct interaction for carbon-carbon pairs in $\mathrm{Fe}-\mathrm{C}$ alloys. That is why as a first approximation we do not consider the direct interaction between carbon-carbon atoms. We do prevent the situation when two carbon atoms occupy the same positions, and, in the present study for describing the C-C interaction, we choose a purely repulsive Born-Mayer potential [19]:

$$
\varphi_{\mathrm{C}-\mathrm{C}}(r)=A \exp \left(-r / r_{\mathrm{s}}\right)
$$

(the energy parameter $A=764.2 \mathrm{eV}$ and the length of screening $r_{\mathrm{s}}=0.219 \AA$ as proposed in [20]) with a cut-off radius of $1.5 \AA$ which is much smaller than the distance between the nearest neighbour carbon atoms.

To initiate the diffusion process, atoms were given initial velocities according to the usual Maxwell distribution at a given temperature, and isothermal annealing procedure was performed in a temperature range of $1273-1373 \mathrm{~K}$ with a step of $50 \mathrm{~K}$. The MD simulations consist of a numerical integration of the equations of atomic motion using a time step $\Delta t=1.5 \times 10^{-15} \mathrm{~s}$ according to the wellknown Verlet algorithm [21]. Periodically, the system was transferred to a state at $T=0 \mathrm{~K}$ where atoms occupied equilibrium positions in a local potential minima. This was done by making use of a static relaxation method. After this, the carbon movements that occurred in the model were analyzed. 


\section{Results and Discussion}

It was found that carbon diffusion in $\mathrm{Fe}_{3} \mathrm{C}$ is realized by means of interstitial sites, which form a base-centered orthorhombic sublattice in cementite with sublattice parameters $a_{\mathrm{I}}=a, b_{\mathrm{I}}=b$ and $c_{\mathrm{I}}=0.5 c$ and 2 sublattice points $(0.0,0.0,0.0)$ and $(0.5,0.5,0.0)$ in units of $a_{\mathrm{I}}, b_{\mathrm{I}}$ and $c_{\mathrm{I}}$ or, in other words, 4 interstitial positions per $\mathrm{Fe}_{3} \mathrm{C}$ unit cell $(0.0,0.0,0.0),(0.5,0.5,0.0),(0.0,0.0,0.5)$ and $(0.5,0.5,0.5)$ in units of $a, b$ and $c$. It should be noted that the distance between the nearest neighbour interstitial sites in [110] and $[1 \overline{1} 0]$ directions (3.404 $\AA$ ) is very close to the distance between the nearest neighbour interstitial sites in the [001] direction (3.372 $\AA$ ). The interstitial sites are less energetically favourable for carbon atoms than their original positions. However, with an increase of temperature, because of the increase in entropy, some fraction $\left(C_{\mathrm{I}}\right)$ of the carbon atoms occupies the interstitial sites and the corresponding same fraction of original carbon positions becomes vacant. In effect, this is equivalent to the formation of Frenkel defects in ionic compounds. In Fig. 1 we report changes of the fraction of the carbon atoms on original positions during MD isothermal annealing at different temperatures. Analysis of the data in Fig.1 shows that in temperature range of 1273-1373 K these fractions approach 'equilibrium' values, $C_{\mathrm{I}}^{\mathrm{eq}}$, after 5-8 ns of the annealing. The temperature dependence of $C_{\mathrm{I}}^{\mathrm{eq}}$ is presented in Fig. 2.

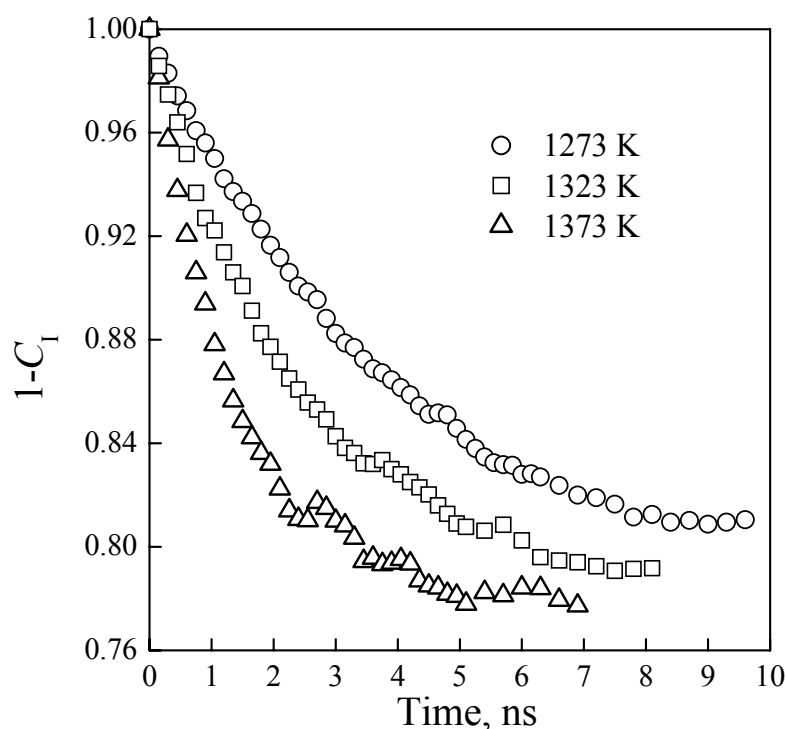

Fig. 1. Changes of the fraction of the carbon atoms on original positions in $\mathrm{Fe}_{3} \mathrm{C}$ during $\mathrm{MD}$ isothermal annealing at different temperatures.

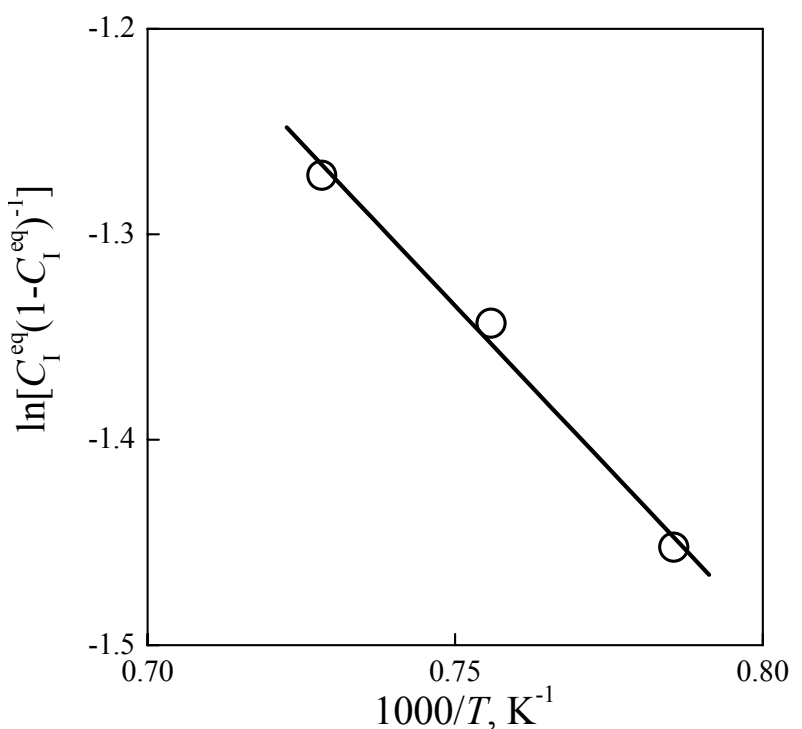

Fig. 2. Temperature dependence of the 'equilibrium' interstitial carbon fraction in $\mathrm{Fe}_{3} \mathrm{C} \mathrm{MD}$ model (points) and its Arrhenius approximation (line).

We give a simple statistical treatment of the dependence of $C_{\mathrm{I}}^{\mathrm{eq}}$ on temperature. The total change in free energy per carbon atom of cementite caused by formation of an interstitial carbon fraction $C_{\mathrm{I}}$ at any temperature $T$, considering the dependence of the interstitial carbon formation free energy on the interstitial carbon fraction $\Delta g_{\mathrm{f}}=\Delta g_{\mathrm{f}}\left(C_{\mathrm{I}}\right)\left(\Delta g_{\mathrm{f}}=E_{\mathrm{f}}-T S_{\mathrm{f}}\right.$, where $E_{\mathrm{f}}$ and $S_{\mathrm{f}}$ are the energy and the entropy of formation of an interstitial carbon), is given by:

$$
\Delta g=\int_{0}^{C_{\mathrm{l}}} \Delta g_{\mathrm{f}}(x) d x-T \Delta s,
$$

where

$$
\Delta s=-2 k\left[\left(1-C_{\mathrm{I}}\right) \ln \left(1-C_{\mathrm{I}}\right)+C_{\mathrm{I}} \ln \left(C_{\mathrm{I}}\right)\right]
$$

is the configurational entropy resulting from the presence of the interstitial carbon ( $k$ is the Boltzmann constant). The multiplier 2 in Eq. (5) appears because there are $\left(1-C_{\mathrm{I}}\right)$ carbon atoms and $C_{\mathrm{I}}$ vacant sites on the original carbon positions as well as the fact that there are $C_{\mathrm{I}}$ carbon atoms and $\left(1-C_{\mathrm{I}}\right)$ vacant sites on the interstitial carbon positions. Upon minimizing the Eq. 4 with respect to the interstitial carbon fraction: 


$$
\left.\frac{\partial \Delta g}{\partial C_{\mathrm{I}}}\right|_{C_{\mathrm{I}}=C_{\mathrm{I}}^{\mathrm{eq}}}=0,
$$

we have that

$$
\frac{C_{\mathrm{I}}^{\mathrm{eq}}}{1-C_{\mathrm{I}}^{\mathrm{eq}}}=\exp \left(-\frac{\Delta g_{\mathrm{f}}^{\mathrm{eq}}}{2 k T}\right),
$$

where $\Delta g_{\mathrm{f}}^{\mathrm{eq}}$ is the interstitial carbon formation free energy at 'equilibrium'. The interstitial carbon formation free energy at 'equilibrium' depends, in general, on the 'equilibrium' interstitial carbon fraction $\Delta g_{\mathrm{f}}^{\mathrm{eq}}=\Delta g_{\mathrm{f}}^{\mathrm{eq}}\left(C_{\mathrm{I}}^{\mathrm{eq}}\right)$. However, in order to crudely estimate the energy $E_{\mathrm{f}}^{\mathrm{eq}}$ and the entropy $S_{\mathrm{f}}^{\mathrm{eq}}$ of formation of an interstitial carbon at 'equilibrium' in the temperature range of 1273-1373 K we will neglect this dependence and consider some averaged values of $E_{\mathrm{f}}^{\mathrm{eq}}$ and $S_{\mathrm{f}}^{\mathrm{eq}}$ in this temperature range. Then, using the Arrhenius approximation of the temperature dependence of the 'equilibrium' interstitial carbon fraction in $\mathrm{Fe}_{3} \mathrm{C} \mathrm{MD}$ model according to Eq. 7 (see Fig. 2), we will have rough estimates of $E_{\mathrm{f}}^{\mathrm{eq}} \approx 0.55 \mathrm{eV} /$ at and $S_{\mathrm{f}}^{\mathrm{eq}} \approx 2 k$ in the temperature range of $1273-1373 \mathrm{~K}$.

The three principal tracer diffusion coefficients $D_{1}^{*}, D_{2}^{*}$ and $D_{3}^{*}$ of carbon atoms along the three orthogonal crystallographic axes $x_{1}, x_{2}$ and $x_{3}$ at each temperature at 'equilibrium' were calculated (Table 2) using the Einstein equation $\left\langle\Delta x_{i}^{2}(t)\right\rangle=2 D_{i}^{*} t$ ( $t$ is time and $\left.i=1,2,3\right)$ from the mean square displacements of the carbon atoms (Fig. 3). Unfortunately, authors do not know any experimental data for carbon tracer diffusion coefficients in $\mathrm{Fe}_{3} \mathrm{C}$ to compare them with the obtained results in Table 2.

Table 2. The principal tracer diffusion coefficients of carbon atoms in $\mathrm{Fe}_{3} \mathrm{C}$ MD model (in $10^{-12} \mathrm{~m}^{2} \mathrm{~s}^{-1}$ ).

\begin{tabular}{|c|c|c|c|}
\hline$T, \mathrm{~K}$ & $D_{1}^{*}$ & $D_{2}^{*}$ & $D_{3}^{*}$ \\
\hline 1273 & 1.5 & 0.9 & 2.2 \\
\hline 1323 & 3.0 & 1.9 & 4.4 \\
\hline 1373 & 4.6 & 2.9 & 6.6 \\
\hline
\end{tabular}

To analyze the results obtained, we first should reveal the most likely movement scenario of carbon atoms in terms of the elementary jumps by the mechanism described above. For this purpose consider the nearest neighbour distances between all possible combinations of carbon original and interstitial positions in the perfect $\mathrm{Fe}_{3} \mathrm{C}$ structure with the lattice parameters given above. It is readily demonstrated that the shortest distance $\left(l^{\prime}=2.547 \AA\right)$ is the distance between the nearest original and interstitial carbon sites. At this distance, every original carbon site has two nearest neighbour interstitial carbon sites. Then, it has two more neighbour interstitial carbon sites at the distance $l^{\prime \prime}=2.657 \AA$. The next two neighbour interstitial carbon sites are located much further; at distance $3.151 \AA$. Absolutely the same situation exists for every interstitial carbon site with respect to the original carbon sites. Other nearest neighbour distances are also considerably larger than the first two $l^{\prime}$ and $l^{\prime \prime}$ : the distance between the nearest neighbour original carbon sites is $3.021 \AA$ and the distance between the nearest neighbour interstitial carbon sites is $3.372 \AA$. Therefore, we believe that carbon diffusion in cementite

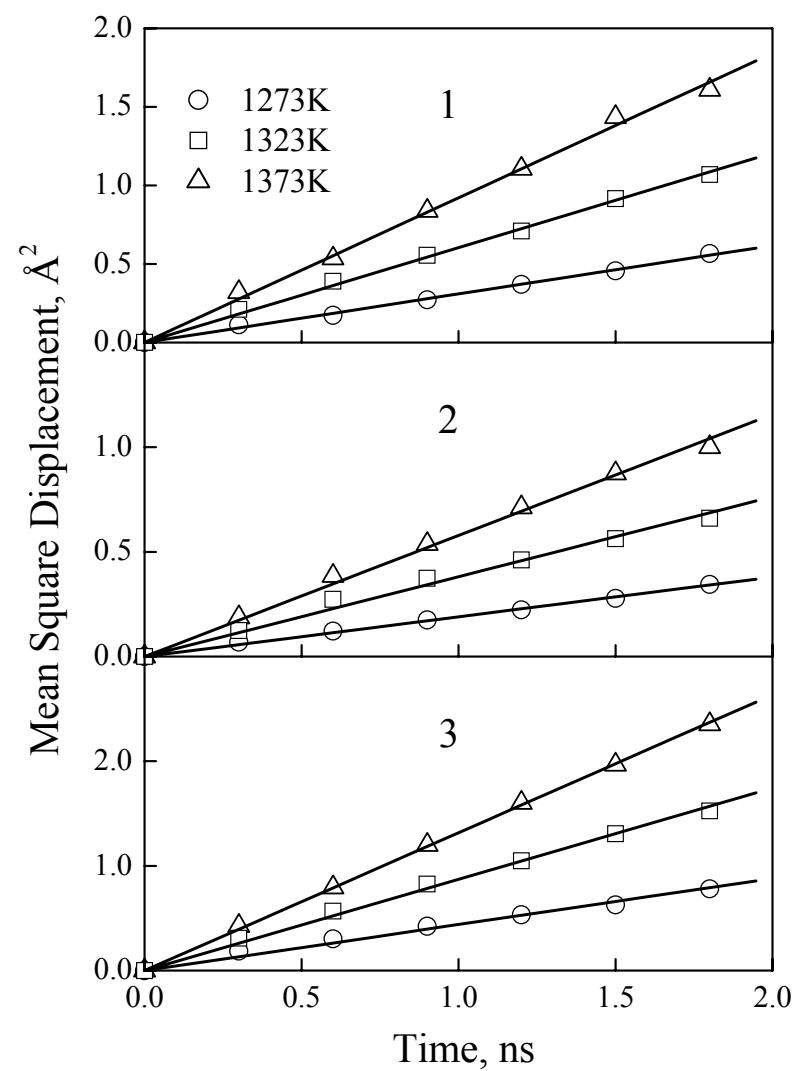

Fig. 3. Mean square displacements of carbon atoms in $\mathrm{Fe}_{3} \mathrm{C}$ along the $x_{1}(1), x_{2}(2)$ and $x_{3}(3)$ principal axes according to the MD simulations. 
comes about predominantly as a consecutive sequence of jumps: original carbon site $\rightarrow$ interstitial carbon site $\rightarrow$ original carbon site $\rightarrow \ldots$ In this case, carbon diffusion in cementite can be described by means of four averaged jump frequencies and two jump distances: $w^{\prime}$ and $w^{\prime \prime}$ are averaged jump frequencies of the transitions original carbon site $\rightarrow$ interstitial carbon site with jump distances $l^{\prime}$ and $l^{\prime \prime}$, respectively; $\widetilde{w}^{\prime}$ and $\widetilde{w}^{\prime \prime}$ are averaged jump frequencies of the transitions interstitial carbon site $\rightarrow$ original carbon site with jump distances $l^{\prime}$ and $l^{\prime \prime}$, respectively. Now, the tracer diffusion coefficients $D_{i}^{*}$ of carbon atoms can be written in a form predicted from random-walk theory: in terms of partial jump frequencies, partial jump distances and partial correlation factors:

$$
D_{i}^{*}=\frac{1}{2}\left\{2\left[\left(1-C_{\mathrm{I}}^{\mathrm{eq}}\right)^{2} w^{\prime}+\left(C_{\mathrm{I}}^{\mathrm{eq}}\right)^{2} \widetilde{w}^{\prime}\right] l_{i}^{\prime 2} f_{i}^{\prime}+2\left[\left(1-C_{\mathrm{I}}^{\mathrm{eq}}\right)^{2} w^{\prime \prime}+\left(C_{\mathrm{I}}^{\mathrm{eq}}\right)^{2} \widetilde{w}^{\prime \prime}\right] l_{i}^{\prime \prime 2} f_{i}^{\prime \prime}\right\}
$$

where $l_{i}^{\prime}, l_{i}^{\prime \prime}$ and $f_{i}^{\prime} f_{i}^{\prime \prime}$ are the projections of the partial jump distances and partial correlation factors along the $x_{i}$ principal axis, respectively. According to detailed balance, at 'equilibrium' we have that:

$$
\left(1-C_{\mathrm{I}}^{\mathrm{eq}}\right)^{2} w^{\prime}=\left(C_{\mathrm{I}}^{\mathrm{eq}}\right)^{2} \widetilde{w}^{\prime}, \quad\left(1-C_{\mathrm{I}}^{\mathrm{eq}}\right)^{2} w^{\prime \prime}=\left(C_{\mathrm{I}}^{\mathrm{eq}}\right)^{2} \widetilde{w}^{\prime \prime} .
$$

Under these conditions, the tracer diffusion coefficients of the carbon atoms are:

$$
D_{i}^{*}=2\left(1-C_{\mathrm{I}}^{\mathrm{eq}}\right)^{2}\left(w^{\prime} l_{i}^{\prime 2} f_{i}^{\prime}+w^{\prime \prime} l_{i}^{\prime \prime 2} f_{i}^{\prime \prime}\right) \text {. }
$$

Next, since $l_{1}^{\prime 2}<<l_{1}^{\prime \prime 2}\left(l_{1}^{\prime}= \pm 0.07 a, l_{1}^{\prime \prime}= \pm 0.43 a\right)$, the jump frequency $w^{\prime \prime}$ can be readily expressed from Eq. 10 for the tracer diffusion coefficient $D_{1}^{*}$ :

$$
w^{\prime \prime} \approx \frac{D_{1}^{*}}{2\left(1-C_{\mathrm{I}}^{\mathrm{eq}}\right)^{2} l_{1}^{\prime \prime 2} f_{1}^{\prime \prime}} .
$$

Unfortunately, we cannot determine directly this jump frequency from the obtained MD data with aid of Eq. 11 because the correlation factor $f_{1}^{\prime \prime}$ is unknown. However, assuming an Arrhenius-type equation for $w^{\prime \prime}=v_{0} \exp \left(S_{\mathrm{m}}^{\prime \prime} / k\right) \exp \left(-E_{\mathrm{m}}^{\prime \prime} / k T\right)\left(v_{0}\right.$ is the effective attempt frequency, $E_{\mathrm{m}}^{\prime \prime}$ and $S_{\mathrm{m}}^{\prime \prime}$ are the activation energy and entropy of the $w^{\prime \prime}$ carbon jump) and neglecting the dependences $f_{1}^{\prime \prime}$, $E_{\mathrm{m}}^{\prime \prime}$ and $S_{\mathrm{m}}^{\prime \prime}$ on $C_{\mathrm{I}}^{\mathrm{eq}}$ in the temperature range $1273-1373 \mathrm{~K}$, we made rough estimations of the activation energy of this jump: $E_{\mathrm{m}}^{\prime \prime} \approx 1.76 \mathrm{eV} / \mathrm{at}$ and the product $v_{0} \exp \left(S_{\mathrm{m}}^{\prime \prime} / k\right) f_{1}^{\prime \prime} \approx 3 \times 10^{14} \mathrm{~s}^{-1}$ using the Arrhenius approximation of the MD data with the aid of Eq. 11 (see Fig. 4).

Finally, using Eq. 10 for the tracer diffusion coefficient $D_{3}^{*}$ together with Eq. 11 and keeping in mind that projections of the jump distances $l^{\prime}$ and $l^{\prime \prime}$ along the $x_{3}$ principal axis are equal $l_{3}^{\prime}=l_{3}^{\prime \prime}=l_{3}= \pm 0.25 \mathrm{c}$, we obtain the expression for

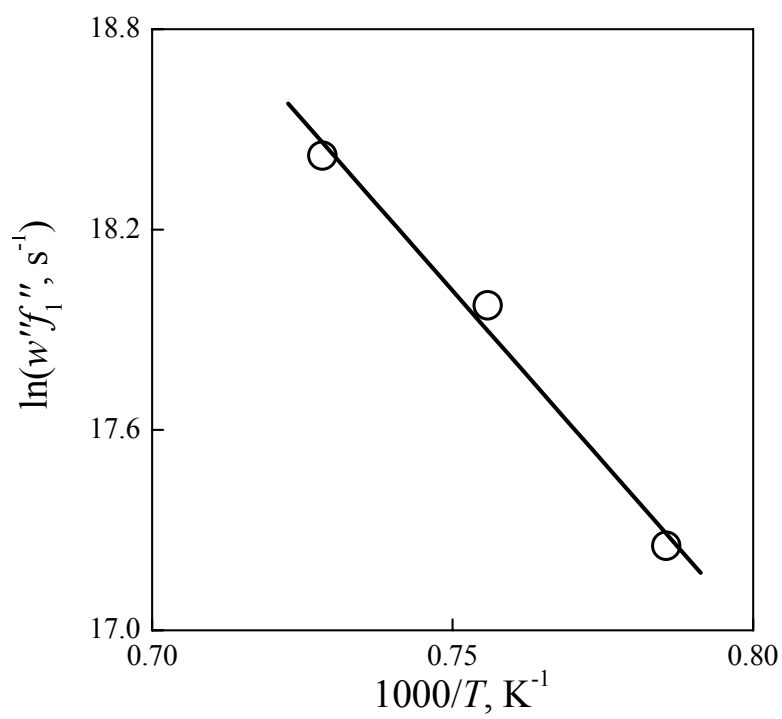

Fig. 4 Arrhenius approximation (line) of the $w^{\prime \prime}$ carbon jump frequency (points) calculated according to Eq. 11 from the MD data $\left(f_{1}^{\prime \prime}\right.$ is the partial correlation factor for the $x_{1}$ principal axis). $w^{\prime}$ in the form:

$$
w^{\prime} \approx w^{\prime \prime} \frac{f_{1}^{\prime \prime}}{f_{3}^{\prime}}\left(\frac{l_{1}^{\prime \prime 2} D_{3}^{*}}{l_{3}^{2} D_{1}^{*}}-\frac{f_{3}^{\prime \prime}}{f_{1}^{\prime \prime}}\right) .
$$


In the temperature range studied, the ratio of the tracer diffusion coefficients $D_{3}^{*} / D_{1}^{*}$ in Eq. 12 according to MD data remains constant $\sim 1.45$, whilst the values of the coefficients vary by a factor of three (see Table 2). Therefore, this form (Eq. 12) emphasises that $w^{\prime}$ differs from $w^{\prime \prime}$ by a constant multiplier, if we neglect the dependences $f_{1}^{\prime \prime}, f_{3}^{\prime}$ and $f_{3}^{\prime \prime}$ on $C_{\mathrm{I}}^{\mathrm{eq}}$ in this temperature range. Consequently, the activation energy of the $w^{\prime}$ carbon jump should be $E_{\mathrm{m}}^{\prime} \approx E_{\mathrm{m}}^{\prime \prime}$.

In conclusion, we should note that for a more accurate analysis of carbon diffusion in cementite the Monte Carlo calculations of the partial correlation factors are essential. These calculations are in progress.

\section{Summary}

We have performed molecular dynamics simulations to investigate carbon diffusion in cementite. The assumption that carbon atoms can interact with each other only indirectly (via neighbouring iron atoms) has been used. We have elucidated the interstitial mechanism of carbon diffusion. Carbon diffusion is realized by means of interstitial sites, which form four positions per $\mathrm{Fe}_{3} \mathrm{C}$ unit cell $(0.0,0.0,0.0),(0.5,0.5,0.0),(0.0,0.0,0.5)$ and $(0.5,0.5,0.5)$ in units of the lattice parameters $a, b$ and $c$. With an increase of temperature a fraction of the carbon atoms occupies the less energetically favourable interstitial sites and the corresponding same fraction of original carbon positions becomes vacant. We have argued that carbon diffusion is predominantly a consecutive chain of jumps: original carbon site $\rightarrow$ interstitial carbon site $\rightarrow$ original carbon site $\rightarrow \ldots$ The principal tracer diffusion coefficients of carbon atoms for this mechanism have been obtained in a form predicted from randomwalk theory. The formation energy $(\sim 0.55 \mathrm{eV} / \mathrm{at})$ of defects (carbon atom on an interstitial position and vacant site on original carbon position) as well as the migration energy $(\sim 1.76 \mathrm{eV} / \mathrm{at})$ of carbon atoms in cementite have been estimated in the temperature range 1273-1373 K from the molecular dynamics data.

\section{Acknowledgements}

This research was supported by the Australian Research Council. One of us (E.V.L.) wishes to thank the University of Newcastle for the award of a Research Fellowship.

\section{References}

[1] M. Hillert, R.D. Sharp: Jernkont. Ann. Vol. 137 (1953), p.785.

[2] B. Ozturk, V.L. Fearing, J.A. Ruth, G.Simkovich: Solid State Ionics Vol. 12 (1984), p. 145.

[3] A. Schneider, G. Inden: CALPHAD Vol. 31 (2007), p. 141.

[4] E. Pippel, J. Woltersdorf, R. Schneider: Mat. Corr. Vol. 49 (1998), p. 309.

[5] P. Szakalos: Mat. Corr. Vol. 54 (2003), p. 752.

[6] H.J. Grabke: Mat. Corr Vol. 54 (2003), p. 736.

[7] D.J. Young: Mat. Sc. Forum Vol. 522-523 (2006), p. 15.

[8] R.Wykoff: Crystal Structures (Interscience, New York 1964).

[9] J. R.A. Johnson: Phys. Rev. Vol.134 (1964), p. A1329.

[10] J.R. Beeler, Jr. and R.A. Johnson: Phys. Rev. Vol. 156 (1967), p. 677.

[11] R.A. Johnson: Phys. Rev. Vol. 145 (1966), p. 423.

[12] R.A. Johnson, G.J. Dienes and A.C. Damask: Acta Metall. Vol. 12 (1964), p. 1215.

[13] R.A. Johnson, A.C. Damask: Acta Metall. Vol 12 (1964), p. 443 (1964).

[14] R.A. Johnson: Acta Metall. Vol.15 (1967), p. 513.

[15] R.A. Johnson: Acta Metall. Vol.13 (1965), p. 1259.

[16] H.M. Pak, M. Doyama: J. Fac.Eng. Univ. Tokyo B Vol. 30 (1969), p. 111.

[17] A.V. Evteev, A.T. Kosilov, and E.V. Levtchenko: Acta Mater. Vol. 51 (2003), p. 2665.

[18] F. Ding, A. Rosen and K. Bolton: J. Chem. Phys. Vol. 121 (2004), p. 2775.

[19] W. Eckstein: Computer Simulation of Ion-Solid Interactions (Springer-Verlag, Berlin 1991).

[20] H.H. Andersen and P. Sigmund: J. Nucl. Instr. Meth. Vol. 38 (1965), p. 238.

[21] L. Verlet: Phys. Rev. Vol. 159 (1967), p. 98. 